\title{
Coefficient bounds for certain classes of analytic functions
}

\author{
by O. P. Juneja (Kanpur), S. Ponnusamy (Helsinki) \\ and S. RAJASEKARAN (Vandalur)
}

\begin{abstract}
We determine coefficient estimates for $\alpha$-spiral functions of order $\varrho$ with respect to $N$-symmetric points $(|\alpha|<\pi / 2,0 \leq \varrho<1$ and $N$ is a positive integer). Sharp coefficient bounds are also obtained for functions of the form $f(z)^{-t}$, where $t$ is a positive integer and $f(z)$ is an $\alpha$-spiral function of order $\varrho$. Using this we deduce coefficient estimates for inverses of univalent $\alpha$-spiral and meromorphic univalent $\alpha$-spiral functions with vanishing early coefficients.
\end{abstract}

1. Introduction. Denote by $\mathcal{H}$ the class of functions

$$
f(z)=z+a_{2} z^{2}+\ldots
$$

which are analytic in the open unit $\operatorname{disc} U=\{z:|z|<1\}$. For a fixed positive integer $N$, let $\mu=e^{2 \pi i / N}$ be the $N$ th root of unity. Then for a function $f \in \mathcal{H}$ given by (1.1), we define

$$
f_{N}(z)=\frac{1}{N} \sum_{j=0}^{N-1} \mu^{-j} f\left(\mu^{j} z\right) .
$$

Using the fact that

$$
\sum_{j=0}^{N-1} \mu^{j k}= \begin{cases}N & \text { if } k \text { is a multiple of } N \\ 0 & \text { otherwise }\end{cases}
$$

it is easily seen that $f_{N}(z)$ has the form

$$
f_{N}(z)=z+a_{N+1} z^{N+1}+a_{2 N+1} z^{2 N+1}+\ldots
$$

A function $f \in \mathcal{H}$ is said to be $\alpha$-spiral of order $\varrho$ with respect to $N$ symmetric points $(|\alpha|<\pi / 2,0 \leq \varrho<1)$ or $N$ - $\alpha$-spiral of order $\varrho$, in

1991 Mathematics Subject Classification: Primary 30C45.

Key words and phrases: subordination, meromorphic univalent and starlike functions.

The final draft of this paper was done with the support of Center of International Mobility. 
symbols $f \in \mathcal{S}_{N}(\alpha, \varrho)$, if

$$
\operatorname{Re}\left\{e^{i \alpha} \frac{z f^{\prime}(z)}{f_{N}(z)}\right\}>\varrho \cos \alpha, \quad z \in U,
$$

where $f_{N}(z)$ is given by $(1.2)$.

The class $\mathcal{S}_{N}(\alpha, \varrho)$ introduced above includes various subclasses studied earlier by a number of workers. For example, $\mathcal{S}_{2}(0,0)$ is the class of starlike functions with respect to symmetric points introduced by Sakaguchi [12] and studied further by Robertson [11]. Similarly, $\mathcal{S}_{N}(0,0)$ is the class of starlike functions with respect to $N$-symmetric points introduced and studied by Chand and Singh [3]. For $N=1, \mathcal{S}_{N}(\alpha, \varrho)$ reduces to the class $\mathcal{S}(\alpha, \varrho)$ of $\alpha$ spiral functions of order $\varrho$ introduced and studied by Libera [6]. Špaček [16] has shown that the family of $\alpha$-spiral functions of order 0 is univalent in $U$. For $\alpha=0, \mathcal{S}_{N}(0, \varrho)\left(\equiv \mathcal{S}_{N}(\varrho)\right)$ leads to the class of starlike functions of order $\varrho$ with respect to $N$-symmetric points. This was introduced and studied by Singh and Singh [15] who claimed to have obtained sharp coefficient estimates for this class. Unfortunately, the claim of Singh and Singh [15] about their coefficient estimates being sharp is not correct. In fact, it can be easily checked that the function given by them is not extremal for the coefficient bounds stated.

In Section 2 of the present paper, we obtain sharp coefficient estimates for the class $\mathcal{S}_{N}(\alpha, \varrho)$ which, for $\alpha=0$, lead to correct and sharp coefficient estimates for the class $\mathcal{S}_{N}^{*}(\varrho)$ introduced by Singh and Singh [15]. For $N=1$, these estimates reduce to sharp coefficient estimates obtained for the class $\mathcal{S}_{N}^{*}(\varrho)$ introduced by Singh and Singh [15]. For $N=1$, these estimates also reduce to sharp coefficient estimates obtained by Libera [6] for the class $\mathcal{S}(\alpha, \varrho)$.

Let $\mathcal{S}(\alpha, \varrho)^{-1}$ denote the class of functions

$$
F(w)=w+A_{2} w^{2}+\ldots
$$

which are inverse to functions in $\mathcal{S}(\alpha, \varrho)$.

Likewise, let $\Sigma_{\alpha}$ denote the class of functions

$$
g(z)=z+b_{0}+b_{1} z^{-1}+\ldots
$$

that are regular and univalent in $V=\{z: 1<|z|<\infty\}$ and satisfy

$$
\operatorname{Re}\left\{e^{i \alpha} \frac{z g^{\prime}(z)}{g(z)}\right\}>0, \quad z \in V .
$$

We observe that $\Sigma_{0} \equiv \Sigma^{*}$, the class of meromorphic univalent starlike functions. Moreover, let $\Sigma_{\alpha}^{-1}$ represent the class of functions which are inverse to functions in $\Sigma_{\alpha}$. 
In [9], MacGregor determined coefficient estimates for starlike functions whose power series representations in $U$ are of the form

$$
f(z)=z+\sum_{n=p+1}^{\infty} a_{n} z^{n} .
$$

Boyd [2] and Srivastava [17] extended MacGregor's result to the classes of starlike functions of order $\varrho\left(\right.$ i.e. $\left.\mathcal{S}^{*}(\varrho)\right)$ and $\mathcal{S}(\alpha, \varrho)$ respectively.

Poole [10] found coefficient estimates for the function $f(z)^{-t}$ when $f \in \mathcal{S}^{*}$ is of the form (1.6) and $t$ is a positive integer. He also obtained coefficient estimates for inverses of functions in $\mathcal{S}^{*}$ and $\Sigma^{*}$ with vanishing early coefficients. Recently, the authors of [1] generalized the results of the above workers by obtaining coefficient bounds for functions of the form $f(z)^{t}$ when $t$ is a positive integer and $f \in \mathcal{S}(\alpha, \varrho)$ is of the form (1.6).

Lately, a number of papers (see e.g. [7], [8], [13], [14]) have appeared in which the coefficient bounds for inverse functions have been determined for the class $\mathcal{S}$ and its various subclasses. A number of techniques have been used to tackle these problems.

In Section 3 of the present paper, we obtain sharp coefficient estimates for functions of the form

$$
f(z)^{-t}=z^{-t}+\sum_{n=-t+p}^{\infty} a_{n}^{(-t)} z^{n}
$$

when $f(z)$, given by (1.6), belongs to $\mathcal{S}(\alpha, \varrho)$ and $t$ is a positive integer. We apply these inequalities to deduce coefficient estimates for functions in $\mathcal{S}(\alpha, 0)^{-1}$ and $\Sigma(\alpha, 0)^{-1}$. The results thus obtained include those of Poole [10] and Juneja and Rajasekaran [5].

We conclude this section by indicating the possibility of extending the notion of $N$-symmetric points for general families of functions considered in [4] and [18] respectively. In fact, Jakubowski [4] discussed a more general family $\mathcal{S}_{m, M}^{*}(\alpha, \varrho)$ of functions $f \in \mathcal{H}$ such that

$$
\left|\left(e^{i \alpha} \frac{z f^{\prime}(z)}{f(z)}-\varrho \cos \alpha-i \sin \alpha\right)[(1-\varrho) \cos \alpha]^{-1}-m\right|<M
$$

Also, he considered a similar definition for a family of meromorphic univalent functions of the form

$$
F(z)=z^{-1}+\sum_{k=1}^{\infty} b_{k} z^{k}, \quad 0<|z|<1,
$$

and proved a sharp estimate for the modulus of the coefficients of functions belonging to these families. 
In [18], Stankiewicz and Waniurski considered the family $\Sigma_{n}^{*}(A, B)$ of meromorphic univalent functions $F$ of the form (1.7) such that

$$
-\frac{z F^{\prime}(z)}{F(z)} \prec \frac{1+A z}{1+B z} \quad(-1 \leq A \leq 1,-A<B \leq 1)
$$

and obtained sharp coefficient estimates for this family.

2. Coefficient bounds for functions in $\mathcal{S}_{N}(\alpha, \varrho)$. In this section we solve the coefficient problem for functions in $\mathcal{S}_{N}(\alpha, \varrho)$. While doing so, we rectify an error of a result in [15].

THEOREM 2.1. If $f(z)=z+\sum_{n=2}^{\infty} a_{n} z^{n} \in \mathcal{S}_{N}(\alpha, \varrho)$, then

$$
\left|a_{m N+1}\right| \leq \prod_{j=0}^{m-1}\left\{\left|\frac{2(1-\varrho) \cos \alpha e^{-i \alpha}}{N}+j\right| /(j+1)\right\}
$$

and

$$
\left|a_{m N+p}\right| \leq \frac{1}{m !} \cdot \frac{N}{m N+p} \prod_{j=0}^{m}\left\{\left|\frac{2(1-\varrho) \cos \alpha e^{-i \alpha}}{N}+j\right|\right\},
$$

for $m=1,2, \ldots$ and $p=2, \ldots, N$. These estimates are sharp for the function $f$ given by

$$
f^{\prime}(z)=\left\{1+\frac{2(1-\varrho) \cos \alpha e^{-i \alpha} z}{1-z}\right\} /\left(1-z^{N}\right)^{2 N^{-1}(1-\varrho) \cos \alpha \exp (-i \alpha)} .
$$

Proof. For $z \in U$ and $f \in \mathcal{S}_{N}(\alpha, \varrho)$, we define

$$
w(z)=\frac{e^{i \alpha}\left(z f^{\prime}(z) / f_{N}(z)-1\right)}{e^{i \alpha} z f^{\prime}(z) / f_{N}(z)+\left(e^{-i \alpha}-2 \varrho \cos \alpha\right)}=\sum_{n=1}^{\infty} w_{n} z^{n} .
$$

Since

$$
\operatorname{Re}\left\{e^{i \alpha} \frac{z f^{\prime}(z)}{f_{N}(z)}\right\}>\varrho \cos \alpha
$$

we have $|w(z)|<1$. We rewrite

$$
f_{N}(z)=z+\sum_{n=2}^{\infty} a_{n} \delta_{n} z^{n}
$$

where

$$
\delta_{n} \equiv \delta(n, N)= \begin{cases}1 & \text { if } n=N p+1, p=1,2, \ldots \\ 0 & \text { otherwise. }\end{cases}
$$

Now replacing $f(z), f_{N}(z), w(z)$ by their power series expansions in the equation (which follows from (2.11))

$$
z f^{\prime}(z)-f_{N}(z)=\left\{z f^{\prime}(z)+\left(e^{-2 i \alpha}-2 \varrho \cos \alpha e^{-i \alpha}\right) f_{N}(z)\right\} w(z),
$$


we obtain

$$
\begin{aligned}
& \sum_{n=2}^{\infty}\left(n-\delta_{n}\right) a_{n} z^{n} \\
& \quad=\left\{\sum_{n=1}^{\infty}\left[n+\left(e^{-2 i \alpha}-2 \varrho \cos \alpha e^{-i \alpha}\right) \delta_{n}\right] a_{n} z^{n}\right\}\left(\sum_{n=1}^{\infty} w_{n} z^{n}\right),
\end{aligned}
$$

where $a_{1}=1$. Comparing the coefficients of $z^{n}$ on both sides of (2.12) we have

$$
\left(n-\delta_{n}\right) a_{n}=\sum_{k=1}^{n-1}\left\{k+\left(e^{-2 i \alpha}-2 \varrho \cos \alpha e^{-i \alpha}\right) \delta_{k}\right\} a_{k} w_{n-k}, \quad n \geq 2 .
$$

This shows that, for $n \geq 2$, the coefficients $a_{n}$ on the left side depend upon the coefficients $a_{1}, \ldots, a_{n-1}$. Therefore, for suitable coefficients $d_{k},(2.12)$ may be rewritten as

$$
\sum_{k=2}^{n}\left(k-\delta_{k}\right) a_{k} z^{k}+\sum_{k=n+1}^{\infty} d_{k} z^{k}=\left\{\sum_{k=1}^{n-1}\left[k+\left(e^{-2 i \alpha}-2 \varrho \cos \alpha e^{-i \alpha}\right) \delta_{k}\right] a_{k} z^{k}\right\} w(z) .
$$

Taking into account that $|w(z)|<1$, and then multiplying each side of this equation by its conjugate, integrating with respect to $\theta$ around 0 to $2 \pi$, and taking the limit as $r \rightarrow 1^{-1}$, we obtain

$$
\begin{aligned}
\sum_{k=2}^{n}\left(k-\delta_{k}\right)^{2}\left|a_{k}\right|^{2} \leq & \left|1+e^{-2 i \alpha}-2 \varrho \cos \alpha e^{-i \alpha}\right|^{2} \\
& +\sum_{k=2}^{n-1}\left|k+\left(e^{-2 i \alpha}-2 \varrho \cos \alpha e^{-i \alpha}\right) \delta_{k}\right|^{2}\left|a_{k}\right|^{2}
\end{aligned}
$$

and so

$$
\begin{aligned}
\left(n-\delta_{n}\right)^{2}\left|a_{n}\right|^{2} \leq & \left|1+e^{-2 i \alpha}-2 \varrho \cos \alpha e^{-i \alpha}\right|^{2} \\
& +\sum_{k=2}^{n-1}\left\{\left|k+\left(e^{-2 i \alpha}-2 \varrho \cos \alpha e^{-i \alpha}\right) \delta_{k}\right|^{2}-\left(k-\delta_{k}\right)^{2}\right\}\left|a_{k}\right|^{2},
\end{aligned}
$$

which after simplification reduces to

$$
\left(n-\delta_{n}\right)^{2}\left|a_{n}\right|^{2} \leq 4(1-\varrho) \cos ^{2} \alpha\left\{(1-\varrho)+\sum_{k=2}^{n-1}(k-\varrho) \delta_{k}\left|a_{k}\right|^{2}\right\},
$$

where we have used the fact that $\delta_{k}=\left(\delta_{k}\right)^{2}$. When $n=N+1$, this gives

$$
\left|a_{N+1}\right| \leq\left|\frac{2(1-\varrho) \cos \alpha e^{-i \alpha}}{N}\right|
$$

which shows that $(2.8)$ is true for $m=1$. 
Now we assume that (2.8) is true for $m=1, \ldots, q-1$. For $m=q$, since the contribution on the right hand side of (2.14) comes only from $a_{N+1}, a_{2 N+1}, \ldots, a_{(q-1) N+1}$, we have

$$
\begin{aligned}
q^{2} N^{2}\left|a_{q N+1}\right|^{2} \leq & 4(1-\varrho) \cos ^{2} \alpha\{(1-\varrho) \\
& \left.+\sum_{j=1}^{q-1}(j N+1-\varrho) \prod_{k=0}^{j-1}\left(\frac{\left|\left[2(1-\varrho) \cos \alpha e^{-i \alpha} / N\right]+k\right|}{k+1}\right)^{2}\right\} \\
= & \left\{q N \prod_{j=0}^{q-1}\left(\frac{\left|\left[2(1-\varrho) \cos \alpha e^{-i \alpha} / N\right]+j\right|}{j+1}\right)\right\}^{2},
\end{aligned}
$$

where the last equality can easily be proved by induction. This completes the proof of (2.8).

When $n=N+p, p=2, \ldots, N,(2.14)$ gives

$$
(N+p)^{2}\left|a_{N+p}\right|^{2} \leq 4(1-\varrho) \cos ^{2} \alpha\left\{(1-\varrho)+(N+1-\varrho)\left|a_{N+1}\right|^{2}\right\},
$$

from which we obtain

$$
\left|a_{N+p}\right| \leq \frac{N}{N+p} \prod_{j=0}^{1}\left|\frac{2(1-\varrho) \cos \alpha e^{-i \alpha}}{N}+j\right| .
$$

Since for $n=m N+p, p=2, \ldots, N$, the contribution on the right hand side of (2.14) comes only from $a_{N+1}, a_{2 N+1}, \ldots, a_{m N+1}$, and so we can use the induction hypothesis as in the proof of (2.8) to obtain

$$
\left|a_{m N+p}\right| \leq \frac{1}{m !} \cdot \frac{N}{m N+p} \prod_{j=0}^{m}\left|\frac{2(1-\varrho) \cos \alpha e^{-i \alpha}}{N}+j\right|,
$$

which is (2.9). This completes the proof of the theorem.

Re mark 2.1. In [15] it was shown that if $\mathcal{S}_{N}^{*}(\varrho)$, then

$$
\left(n-\delta_{n}\right)^{2}\left|a_{n}\right|^{2} \leq 4(1-\varrho)^{2}+2 \sum_{k=2}^{n-1}\{k+(1-\varrho)(k-2 \varrho)\} \delta_{k}\left|a_{k}\right|^{2} .
$$

It was further claimed that the estimate (2.15) is sharp for the function (2.10) when $\alpha=0$. However, it is easy to check that this claim is not correct for any $n>2 N$. In fact, when $\alpha=0$, for the function $f$ given by (2.10),

$$
\begin{aligned}
(2 N)^{2}\left|a_{2 N+1}\right|^{2} & =\left\{(2-2 \varrho)\left(\frac{2-2 \varrho}{N}+1\right)\right\}^{2} \\
& <4(1-\varrho)^{2}+2\{(N+1)+(1-\varrho)(N+1-2 \varrho)\} \frac{(2-2 \varrho)^{2}}{N^{2}}
\end{aligned}
$$


and

$$
\begin{aligned}
(2 N & +p)^{2}\left|a_{2 N+p}\right|^{2} \\
= & \left\{\frac{N}{2 !} \prod_{j=0}^{2}\left(\frac{2-2 \varrho}{N}+j\right)\right\}^{2} \\
< & 4(1-\varrho)^{2}+2\{(N+1)+(1-\varrho)(N+1-2 \varrho)\} \frac{(2-2 \varrho)^{2}}{N^{2}} \\
& +2\{2 N+1+(1-\varrho)(2 N+1-\varrho)\} \frac{\{((2-2 \varrho) / N)((2-2 \varrho) / N+1)\}^{2}}{(2 !)^{2}}
\end{aligned}
$$

where $p=2, \ldots, N$, and so on.

On the other hand, our estimate (2.14), for $\alpha=0$, leads to

$$
\left(n-\delta_{n}\right)^{2}\left|a_{n}\right|^{2} \leq 4(1-\varrho)\left\{(1-\varrho)+\sum_{k=2}^{n-1}(k-\varrho) \delta_{k}\left|a_{k}\right|^{2}\right\}
$$

and it is easy to see that equality occurs for every $n$ in (2.16) for the function $f$ given by $(2.10)$ when $\alpha=0$.

Remark 2.2. Singh and Singh [15] have defined the class $\mathcal{C}_{N}(\varrho)$ as follows: $f \in \mathcal{C}_{N}(\varrho)$ if and only if $z f^{\prime} \in \mathcal{S}_{N}^{*}(\varrho)$. For $f(z)=z+\sum_{n=2}^{\infty} a_{n} z^{n} \in$ $\mathcal{C}_{N}(\varrho)$, they have obtained the estimates

$$
\begin{aligned}
& \{m N(m N+1)\}^{2}\left|a_{m N+1}\right|^{2} \\
\leq & 4(1-\varrho)^{2}+2 \sum_{k=1}^{m-1}\{(2-\varrho)(k N+1)-2 \varrho(1-\varrho)\}(k N+1)^{2}\left|a_{k N+1}\right|^{2}
\end{aligned}
$$

for $m=1,2, \ldots$, and

$$
\begin{aligned}
& (m N+p)^{2}\left|a_{m N+p}\right|^{2} \\
& \leq 4(1-\varrho)^{2}+\sum_{k=1}^{m}\{(2-\varrho)(k N+1)-2 \varrho(1-\varrho)\}(k N+1)^{2}\left|a_{k N+1}\right|^{2}
\end{aligned}
$$

for $m=0,1, \ldots$ and $p=2, \ldots, N$.

In view of (2.16), the results (2.17) and (2.18) can be modified to

$$
\begin{aligned}
\{m N(m N & +1)\}^{2}\left|a_{m N+1}\right|^{2} \\
& \leq 4(1-\varrho)\left\{(1-\varrho)+\sum_{k=1}^{m-1}(k N+1-\varrho)(k N+1)^{2}\left|a_{k N+1}\right|^{2}\right\}
\end{aligned}
$$

for $m=1, \ldots$, and 


$$
\begin{aligned}
& (m N+p)^{4}\left|a_{m N+p}\right|^{2} \\
& \quad \leq 4(1-\varrho)\left\{(1-\varrho)+\sum_{k=1}^{m-1}(k N+1-\varrho)(k N+1)^{2}\left|a_{k N+1}\right|^{2}\right\}
\end{aligned}
$$

for $m=0,1, \ldots$ and $p=2, \ldots, N$.

Now, putting $\alpha=0$, Theorem 2.1 gives the following result, which is a refinement of the results in [3] and [15].

Corollary 2.1. If $f(z)=z+\sum_{n=2}^{\infty} a_{n} z^{n} \in \mathcal{S}_{N}^{*}(\varrho)$, then

$$
\left|a_{m N+1}\right| \leq \prod_{j=0}^{m-1}\left\{\left(\frac{2-2 \varrho}{N}+j\right) /(j+1)\right\}
$$

for $m=1,2, \ldots$, and

$$
\left|a_{m N+p}\right| \leq \frac{1}{m !} \cdot \frac{N}{m N+p} \prod_{j=0}^{m}\left(\frac{2-2 \varrho}{N}+j\right)
$$

for $m=0,1, \ldots$ and $p=2, \ldots, N$. These estimates are sharp.

In view of the fact that $f \in \mathcal{C}_{N}(\varrho)$ if and only if $z f^{\prime} \in \mathcal{S}_{N}^{*}(\varrho)$, we have

Corollary 2.2. If $f(z)=z+\sum_{n=2}^{\infty} a_{n} z^{n} \in \mathcal{C}_{N}(\varrho)$, then

$$
\left|a_{m N+1}\right| \leq \frac{1}{m N+1} \prod_{j=0}^{m-1}\left\{\left(\frac{2-2 \varrho}{N}+j\right) /(j+1)\right\}
$$

for $m=1,2, \ldots$, and

$$
\left|a_{m N+p}\right| \leq \frac{1}{m !} \cdot \frac{N}{(m N+p)^{2}} \prod_{j=0}^{m}\left(\frac{2-2 \varrho}{N}+j\right)
$$

for $m=0,1, \ldots$ and $p=2, \ldots, N$. These inequalities are sharp.

\section{Some other coefficient bounds}

TheOREM 3.1. Suppose $f(z)=z+\sum_{n=p+1}^{\infty} a_{n} z^{n} \in \mathcal{S}(\alpha, \varrho)$, and for integral $t \geq 1$, let

$$
f(z)^{-t}=z^{-t}+\sum_{\gamma=-t+p}^{\infty} a_{\gamma}^{(-t)} z^{\gamma}, \quad 0<|z|<1 .
$$

Then

$$
\left|a_{\gamma}^{(-t)}\right| \leq \frac{m p}{\gamma+t} \prod_{j=0}^{m-1}\left(\frac{\left|(2 t / p)(1-\varrho) \cos \alpha e^{-i \alpha}-j\right|}{j+1}\right)
$$


for $-t+m p \leq \gamma \leq-t+(m+1) p-1, m=1, \ldots, M+1$, where $M=$ $[t(1-\varrho) / p]$. (Here $[x]$ denotes the largest integer not exceeding $x$.$) The$ result is sharp for $\gamma=-t+m p$.

The following two lemmas will be useful in the proof of this theorem.

Lemma 3.1. If $p, t$ and $q$ are positive integers, then for $0 \leq \varrho<1$, we have

$$
\begin{aligned}
4 t(1-\varrho) & \cos ^{2} \alpha\{t(1-\varrho) \\
& \left.+\sum_{m=1}^{q-1}[t(1-\varrho)-m p] \prod_{j=0}^{m-1}\left(\frac{\left|(2 t / p)(1-\varrho) \cos \alpha e^{-i \alpha}-j\right|}{j+1}\right)^{2}\right\} \\
= & (q p)^{2}\left\{\prod_{j=0}^{q-1}\left(\frac{\left|(2 t / p)(1-\varrho) \cos \alpha e^{-i \alpha}-j\right|}{j+1}\right)\right\}^{2}
\end{aligned}
$$

whenever $t(1-\varrho)-(q-1) p \geq 0$.

The above lemma can be easily proved by induction on $q$.

Lemma 3.2. If $p, t$ and $q$ are positive integers and $0 \leq \varrho<1$, then

$$
(\gamma+t)^{2} \geq \frac{(q p)^{2}(-\gamma-\varrho t)}{t(1-\varrho)-q p}
$$

for $\gamma \geq-t+q p$ and $t(1-\varrho)-(q-1) p \geq 0$.

The proof of Lemma 3.2 is straightforward and hence omitted.

Proof of Theorem 3.1. For $0<|z|<1$, let

$$
q(z)=\frac{e^{i \alpha}}{-t} \cdot \frac{z\left[f(z)^{-t}\right]^{\prime}}{f(z)^{-t}}=e^{i \alpha} \frac{z f^{\prime}(z)}{f(z)}
$$

and $q(0)$ can be defined so that $q(z)$ is continuous at $z=0$. Moreover, let

$$
w(z)=\frac{q(z)-e^{i \alpha}}{q(z)+e^{-i \alpha}-2 \varrho \cos \alpha}=\sum_{n=p}^{\infty} w_{n} z^{n} .
$$

Since $\operatorname{Re} q(z)>\varrho \cos \alpha$, we have $|w(z)|<1$ in $|z|<1$. Equating the coefficients of the same powers on both sides of (3.20) we get

$$
e^{i \alpha}\left\{\left[f(z)^{-t}\right]^{\prime}+t f(z)^{-t}\right\}=\left\{e^{i \alpha} z\left[f(z)^{-t}\right]^{\prime}-\left(e^{-i \alpha}-2 \varrho \cos \alpha\right) t f(z)^{-t}\right\} w(z)
$$


or

$$
\begin{aligned}
e^{i \alpha} \sum_{n=t+p}^{\infty}(n+t) & a_{n}^{(-t)} z^{n} \\
= & \left(\sum_{n=p}^{\infty} w_{n} z^{n}\right)\left\{-2 t(1-\varrho) \cos \alpha z^{-t}\right. \\
& \left.+\sum_{n=-t+p}^{\infty}\left[n e^{i \alpha}-t\left(e^{-i \alpha}-2 \varrho \cos \alpha\right)\right] a_{n}^{(-t)} z^{n}\right\},
\end{aligned}
$$

from which we obtain the relations

$$
e^{i \alpha} \gamma a_{\gamma-t}^{(-t)}=-2 t(1-\varrho) \cos \alpha w_{\gamma}, \quad \gamma=p, p+1, \ldots, 2 p-1 .
$$

Since $|w(z)|<1$, we have $\sum_{n=p}^{\infty}\left|w_{n}\right|^{2} \leq 1$ and therefore

$$
\sum_{n=p}^{2 p-1}\left|w_{n}\right|^{2} \leq 1 \text {. }
$$

Now (3.22) and (3.23) yield

$$
\sum_{n=p}^{2 p-1} \gamma^{2}\left|a_{\gamma-t}\right|^{2} \leq 4 t^{2}(1-\varrho)^{2} \cos ^{2} \alpha .
$$

We rewrite (3.21) in the form

$$
\begin{aligned}
\sum_{n=-t+p}^{k} e^{i \alpha}(n+t) & a_{n}^{(-t)} z^{n}+\sum_{n=k+1}^{\infty} d_{n} z^{n} \\
= & w(z)\left\{-2 t(1-\varrho) \cos \alpha z^{-t}\right. \\
& \left.+\sum_{n=-t+p}^{k-p}\left[n e^{i \alpha}-t\left(e^{-i \alpha}-2 \varrho \cos \alpha\right)\right] a_{n}^{(-t)} z^{n}\right\} .
\end{aligned}
$$

Taking into account that $|w(z)|<1$, and then multiplying each side of (3.25) by its conjugate, integrating around $|z|=r<1$ and letting $r \rightarrow 1^{-}$yields the inequality

$$
\begin{aligned}
& \sum_{n=-t+p}^{k-p}(n+t)^{2}\left|a_{n}^{(-t)}\right|^{2} \\
& \quad \leq 4 t(1-\varrho) \cos ^{2} \alpha\left\{t(1-\varrho)+\sum_{n=-t+p}^{k-p}(-n-\varrho t)\left|a_{n}^{(-t)}\right|^{2}\right\} .
\end{aligned}
$$


Putting $k=-t+(q+1) p-1,(3.25)$ can be rewritten as

$$
\begin{aligned}
& \sum_{n=-t+p}^{-t+(q+1) p-1}(n+t)^{2}\left|a_{n}^{(-t)}\right|^{2} \\
& \quad \leq 4 t(1-\varrho) \cos ^{2} \alpha\left\{t(1-\varrho)+\sum_{n=-t+p}^{-t+q p-1}(-n-\varrho t)\left|a_{n}^{(-t)}\right|^{2}\right\} \\
& \quad=4 t(1-\varrho) \cos ^{2} \alpha\left\{t(1-\varrho)+\sum_{m=1}^{q-1} \sum_{n=-t+m p}^{-t+(m+1) p-1}(-n-\varrho t)\left|a_{n}^{(-t)}\right|^{2}\right\}
\end{aligned}
$$

Now, by Lemma 3.2 and (3.24),

$$
\begin{aligned}
\sum_{n=-t+p}^{-t+2 p-1}(-n-\varrho t)\left|a_{n}^{(-t)}\right|^{2} & =\frac{t(1-\varrho)-p}{p^{2}} \sum_{n=-t+p}^{-t+2 p-1} \frac{p^{2}(-n-\varrho t)}{t(1-\varrho)-p}\left|a_{n}^{(-t)}\right|^{2} \\
& \leq \frac{[t(1-\varrho)-p]}{p^{2}} \sum_{n=-t+p}^{-t+2 p-1}(n+t)^{2}\left|a_{n}^{(-t)}\right|^{2}, \\
& \leq \frac{[t(1-\varrho)-p]}{p^{2}} \cdot 4 t^{2}(1-\varrho)^{2} \cos ^{2} \alpha, \\
& =[t(1-\varrho)-p]\left|(2 t / p)(1-\varrho) \cos \alpha e^{i \alpha}\right|^{2} .
\end{aligned}
$$

Now we assume

$$
\begin{aligned}
\sum_{n=-t+m p}^{-t+(m+1) p-1}(- & n-\varrho t)\left|a_{n}^{(-t)}\right|^{2} \\
& \leq[t(1-\varrho)-m p] \prod_{j=0}^{m-1}\left(\frac{\left|(2 t / p)(1-\varrho) \cos \alpha e^{-i \alpha}-j\right|}{j+1}\right)^{2}
\end{aligned}
$$

for $m=2, \ldots, q-1$. Then from (3.26),

$$
\begin{aligned}
& \sum_{n=-t+q p}^{-t+(q+1) p-1}(n+t)^{2}\left|a_{n}^{(-t)}\right|^{2} \\
& \quad \leq 4 t(1-\varrho) \cos ^{2} \alpha\left\{t(1-\varrho)+\sum_{m=1}^{q-1} \sum_{n=-t+m p}^{-t+(m+1) p-1}(-n-\varrho t)\left|a_{n}^{(-t)}\right|^{2}\right\} \\
& \quad=4 t(1-\varrho) \cos ^{2} \alpha\left\{t(1-\varrho)+\sum_{m=1}^{q-1}[t(1-\varrho)-m p]\right.
\end{aligned}
$$




$$
\begin{gathered}
\left.\times \prod_{j=0}^{m-1}\left(\frac{\left|(2 t / p)(1-\varrho) \cos \alpha e^{-i \alpha}-j\right|}{j+1}\right)^{2}\right\} \\
=(q p)^{2} \prod_{j=0}^{q-1}\left(\frac{\left|(2 t / p)(1-\varrho) \cos \alpha e^{-i \alpha}-j\right|}{j+1}\right)^{2},
\end{gathered}
$$

by Lemma 3.1, as long as $t(1-\varrho)-(q-1) p \geq 0$. Now we use Lemma 3.2 and this estimate to show that

$$
\begin{aligned}
\sum_{n=-t+q p}^{-t+(q+1) p-1}(- & n-\varrho t)\left|a_{n}^{(-t)}\right|^{2} \\
& =\frac{[t(1-\varrho)-q p]}{(q p)^{2}}\left\{\sum_{n=-t+q p}^{-t+(q+1) p-1} \frac{(q p)^{2}(-n-\varrho t)}{t(1-\varrho)-q p}\left|a_{n}^{(-t)}\right|^{2}\right\} \\
& \leq \frac{[t(1-\varrho)-q p]}{(q p)^{2}}\left\{\sum_{n=-t+q p}^{-t+(q+1) p-1}(n+t)^{2}\left|a_{n}^{(-t)}\right|^{2}\right\} \\
& =[t(1-\varrho)-q p] \prod_{j=0}^{q-1}\left(\frac{\left|(2 t / p)(1-\varrho) \cos \alpha e^{-i \alpha}-j\right|}{j+1}\right)^{2} .
\end{aligned}
$$

Thus we have established by induction that

$$
\begin{aligned}
& \sum_{n=-t+m p}^{-t+(m+1) p-1}(n+t)^{2}\left|a_{n}^{(-t)}\right|^{2} \\
& \quad \leq(m p)^{2} \prod_{j=0}^{m-1}\left(\frac{\left|(2 t / p)(1-\varrho) \cos \alpha e^{-i \alpha}-j\right|}{j+1}\right)^{2}
\end{aligned}
$$

and

$$
\begin{aligned}
\sum_{n=-t+m p}^{-t+(m+1) p-1}( & n-\varrho t)\left|a_{n}^{(-t)}\right|^{2} \\
& \leq[t(1-\varrho)-m p] \prod_{j=0}^{m-1}\left(\frac{\left|(2 t / p)(1-\varrho) \cos \alpha e^{-i \alpha}-j\right|}{j+1}\right)^{2}
\end{aligned}
$$

for $m$ such that $t(1-\varrho)-(m-1) p \geq 0$.

The theorem now follows from (3.27). If $n=-t+m p$, the inequality (3.19) is sharp for the function

$$
f(z)=z\left(1-z^{p}\right)^{-2(1-\varrho) \cos \alpha \exp (-i \alpha) / p} .
$$

This completes the proof. 
Now take two functions

$F(w)=w+\sum_{n=2}^{\infty} A_{n} w^{n} \in \mathcal{S}(\alpha, 0)^{-1}, \quad G(w)=w+\sum_{n=1}^{\infty} B_{n} w^{-n} \in \Sigma(\alpha, 0)^{-1}$.

It has been proved in [5] that

$$
A_{n}=a_{-1}^{(-n)} / n \quad \text { and } \quad B_{n}=-a_{1}^{(-n)} / n, \quad \text { for } n=1,2, \ldots
$$

Now using these results and Theorem 3.1, we obtain the following results.

Theorem 3.2. If $F(w)=w+\sum_{n=p+1}^{\infty} A_{n} w^{n} \in \mathcal{S}(\alpha, 0)^{-1}$, then

$$
\left|A_{n}\right| \leq \frac{m p}{n(n-1)} \prod_{j=0}^{m-1}\left(\frac{\left|(2 n / p) \cos \alpha e^{-i \alpha}-j\right|}{j+1}\right)
$$

for $m p+1 \leq n \leq(m+1) p, m=1,2, \ldots$

Theorem 3.3. If $G(w)=w+\sum_{n=p-1}^{\infty} B_{n} w^{-n} \in \Sigma(\alpha, 0)^{-1}$, then

$$
\left|B_{n}\right| \leq \frac{m p}{n(n+1)} \prod_{j=0}^{m-1}\left(\frac{\left|(2 n / p) \cos \alpha e^{-i \alpha}-j\right|}{j+1}\right)
$$

for $m p-1 \leq n \leq(m+1) p-2, m=1,2, \ldots$

For $\alpha=0$, Theorems 3.2 and 3.3 give the following results of Poole [10].

Corollary 3.1. If $F(w)=w+\sum_{n=p+1}^{\infty} A_{n} w^{n} \in \mathcal{S}^{*-1}$, then

$$
\left|A_{n}\right| \leq \frac{m p}{n(n-1)} \prod_{j=0}^{m-1}\left(\frac{(2 n / p)-j}{j+1}\right)
$$

for $m p+1 \leq n \leq(m+1) p, m=1,2, \ldots$

Corollary 3.2. If $G(w)=w+\sum_{n=p-1}^{\infty} B_{n} w^{-n} \in \Sigma^{*-1}$, then

$$
\left|B_{n}\right| \leq \frac{m p}{n(n+1)} \prod_{j=0}^{m-1}\left(\frac{(2 n / p)-j}{j+1}\right)
$$

for $m p-1 \leq n \leq(m+1) p, m=1,2, \ldots$

Moreover, when $n=m p+1$, Theorem 3.2 gives a result in [5]. Similarly, when $n=m p-1$, Theorem 3.3 gives a result in [5].

\section{References}

[1] B. L. Bhatia and S. Rajasekaran, Coefficient estimates for alpha-spiral functions, Bull. Austral. Math. Soc. 28 (1983), 319-329.

[2] A. V. Boyd, Coefficient estimates for starlike functions of order $\alpha$, Proc. Amer. Math. Soc. 17 (1966), 1016-1019. 
[3] R. Chand and P. Sing h, On certain schlicht mappings, Indian J. Pure Appl. Math. 10 (1979), 1167-1174.

[4] Z. J. Jakubowski, On the coefficients of starlike functions of some classes, Bull. Acad. Polon. Sci. 9 (1971), 811-815.

[5] O. P. Juneja and S. Rajasekaran, Coefficient estimates for inverses of $\alpha$-spiral functions, Complex Variables Theory Appl. 6 (1986), 99-108.

[6] R. J. Libera, Univalent $\alpha$-spiral functions, Canad. J. Math. 19 (1967), 449-456.

[7] R. J. Libera and E. J. Złotkiewicz, Early coefficients of the inverse of a regular convex function, Proc. Amer. Math. Soc. 85 (1982), 225-230.

[8] - - - Coefficient bounds for the inverse of odd univalent functions, Complex Variables Theory Appl. 3 (1983), 185-189.

[9] T. H. MacGregor, Coefficient estimates for starlike mappings, Michigan Math. J. 10 (1963), 277-281.

[10] J. T. Poole, On starlike functions, Proc. Amer. Math. Soc. 19 (1968), 495-500.

[11] M. S. Robertson, Applications of the subordination principle to univalent functions, Pacific J. Math. 11 (1961), 315-324.

[12] K. Sakaguchi, On a certain univalent mapping, J. Math. Soc. Japan 11 (1959), $72-75$.

[13] G. Schober, Coefficients of inverses of meromorphic univalent functions, Proc. Amer. Math. Soc. 67 (1977), 111-116.

[14] _, Coefficient estimates for the inverses of schlicht functions, in: Aspects of Contemporary Complex Analysis, Academic Press, New York, 1980, 503-513.

[15] P. Singh and P. Singh, Integral representations of functions in certain classes of univalent functions, Indian J. Pure Appl. Math. 12 (1981), 459-471.

[16] L. Špaček, Contributions à la théorie des fonctions univalentes, Časopis Pěst. Mat. 62 (1933), 12-19 (in Czech).

[17] R. S. L. Srivastava, Univalent spiral functions, in: Topics in Analysis, Lecture Notes in Math. 419, Springer, Berlin, 1974, 327-341.

[18] J. Stankiewicz and J. Waniurski, Some classes of functions subordinate to linear transformation and their applications, Ann. Univ. M. Curie-Skłodowska 28 (9) (1974), 85-94.

DEPARTMENT OF MATHEMATICS

INDIAN INSTITUTE OF TECHNOLOGY

KANPUR-208 016, INDIA

DEPARTMENT OF MATHEMATICS

CRESCENT ENGINEERING COLLEGE

VANDALUR

MADRAS-600 048, INDIA
DEPARTMENT OF MATHEMATICS UNIVERSITY OF HELSINKI P.O. BOX 4, HALLITUSKATU 15 FIN-00014 HELSINKI, FINLAND 